\title{
Throughput Enhancement in TDMA through Carrier Interferometry Pulse Shaping
}

\author{
Balasubramaniam Natarajan \\ RAWCom Lab, Dept. of ECE \\ Colorado State University \\ Fort Collins, CO - 80523-1373 \\ nbalsu@engr.colostate.edu
}

\author{
Carl R.Nassar \\ RAWCom Lab, Dept. of ECE \\ Colorado State University \\ Fort Collins, CO - 80523-1373 \\ carln@engr.colostate.edu
}

\author{
Steve Shattil \\ Idris Communications \\ 4980 Meredith Way \\ Boulder, CO-80303 \\ temporal@dimensional.com
}

\begin{abstract}
This paper introduces a novel TDMA scheme that provides enhanced throughput by employing carrier interferometry pulse shapes (CI pulse shapes). At the transmitter, CI pulse shapes are created from the superposition of $N$ carriers, which generates a short mainlobe (pulse) in time. CI pulse shapes are positioned both orthogonally and pseudo-orthogonally in time, enabling the introduction of additional bits into a TDMA burst. Specifically, up to a $100 \%$ increase in throughput can be achieved. At the receiver, a novel TDMA detector is deployed where: the pulse shape is broken down into its frequency components and optimally recombined to create frequency diversity benefits. Simulations performed over hilly terrain $(H T)$ and typical urban (TU) GSM channel models indicate that, with a 100\% increase in throughput, the proposed system offers up to 6.5 $d B$ performance gains at probability of error of $10^{-2}$ relative to a standard GSM system employing a decision feedback receiver.
\end{abstract}

\section{Introduction}

Time division multiple access (TDMA) is a popular technology in mobile communication systems worldwide [1]. One example of a wireless system that employs a TDMA architecture is the Global system for Mobile Communications (GSM) [2], which is one of the most successful digital cellular systems in the world.

GSM systems employ Gaussian pulse shaping and GMSK modulation to gain in spectral efficiency. These systems experience intersymbol interference (ISI) due to multipath. To maintain acceptable performance in the presence of ISI, GSM systems may employ a decision feedback equalizer (DFE) receiver (e.g.,[3]). The DFE receiver provides significant reduction in complexity when compared to a maximum likelihood sequence estimator (MLSE) while suffering only a modest performance degradation ([2],[4]).

In this paper we introduce a novel pulse shaping method called carrier interferometry pulse shaping (CI pulse shaping) into the TDMA architecture. Here, the pulse shape is composed of $N$ carriers equally spaced in frequency. The carriers combine to ensure a pulse shape corresponding to a mainlobe in the time domain (with sidelobe activity). These pulse shapes can be overlaped in time without destroying orthogonality. Furthermore, additional pulse shapes can be accommodated in the same time slot by allowing for a pseudo orthogonal overlap of pulse shapes in time. In this way, assigning one bit or symbol per pulse shape, it is possible to nearly double throughput with no extra expense in bandwidth or burst duration, and without the cost of performance degradation as seen later.

Novel receiver designs which exploit frequency diversity ensure that the BER performance of CI/TDMA is better than that of GSM employing a DFE, even when throughput is doubled over traditional GSM.

Section 2 briefly reviews typical TDMA frame structures and the GSM system. Section 3 introduces the novel CI pulse shaping method and the new TDMA transmitter. It explains increased capacities through pseudo orthogonal positioning of $\mathrm{CI}$ pulse shapes in time. Section 4 presents the receiver structure and Section 5 discusses the channel model used. Finally, in Section 6, the performance results are provided.

\section{Overview of TDMA/GSM}

TDMA systems divide the radio spectrum into time slots of duration $T_{s}$, and each user is allowed to transmit $N$ bits $\left(T_{s}=N \cdot T_{b}\right)$ in a slot. All user slots combine together to create a TDMA frame.

In the popular GSM system, each user slot contains $N$ $=148$ bits. Bit rate is $270.8 \mathrm{kbits} / \mathrm{s}$, bit duration $T_{b}$ is 
$3.69 \mu \mathrm{s}$, and slot time $T_{8}=576.6 \mu \mathrm{s}$ (with $30.44 \mu$ s allocated as guard time). GSM systems use binary Gaussian minimum shift keying (GMSK) modulation. This is characterized by a constant envelope and narrow bandwidth, and information is carried on the phase of the transmitted signal. Specifically, the information bits of user $p$ are first differentially encoded, producing an NRZ (nonreturn-to-zero) symbol stream $\mathrm{d}_{p}=d_{p}(i)(i=1,2, \ldots, N)$; Next this symbol stream excites a transmit filter $h(t)$ with a Gaussian impulse response. The waveform at the output of the Gaussian filter may be expressed as

$$
\psi_{p}(t)=\sum_{i=1}^{N} d_{p}(i) q\left(t-i T_{b}\right)
$$

where

$$
q(t)=\int_{0}^{T_{b}} h(t-\tau) m(\tau) d \tau
$$

Here, $m(\tau)$ is the rectangular waveform of the NRZ pulse and $h(t)$ is the Gaussian pulse defined by

$$
h(t)=\frac{\eta B}{\sqrt{\pi}} e^{-(\eta B t)^{2}} .
$$

$B$ is the $3 \mathrm{~dB}$ bandwidth $\left(B T_{b}=0.3\right.$ for GSM), and $\eta=$ $\pi / \sqrt{2 \ln 2} \approx 2.668$. Finally, the Gaussian filter output $\psi_{p}(t)$ is integrated and this serves as the phase of the transmitted waveform. The complex baseband representation of the output signal corresponds to

$$
s_{p}(t)=e^{j \phi_{p}(t)} .
$$

where $\phi_{p}(t)=\frac{\pi}{2} \int_{-\infty}^{t} \psi_{p}(t) d t$. Typically, the non-linear baseband GMSK signal is approximated by a linear modulation[6] to aid in system analysis, receiver design and simulation.

\section{Transmitter Structure for CITDMA}

Figure 1 shows the creation of the new carrier interferometry pulse shape ( $\mathrm{CI}$ pulse shape). As seen in figure, the pulse is created by superpositioning $N$ carriers equally spaced in frequency by $\Delta f=1 / T_{s}$ ( $N$ corresponds to the number of bits per slot, e.g., 148 in GSM). This choice of $\Delta f$ assures that the total available bandwidth is the same as in the GSM system (Figure 2). The output pulse shape, which can be implemented using FFT's, corresponds mathematically to

$$
h(t)=\sum_{i=1}^{N} A \cos (i 2 \pi \Delta f t)
$$

Using properties of summation and sinusoids, the pulse shape corresponds to

$$
h(t)=A \cdot \frac{\sin \left(\frac{N}{2} 2 \pi \Delta f t\right)}{\sin \left(\frac{1}{2} 2 \pi \Delta f t\right)} \cdot \cos \left(\frac{N+1}{2} 2 \pi \Delta f t\right)
$$

Here, $A=\sqrt{\frac{1}{N}} \sqrt{\frac{2}{T_{s}}}$ is a constant that ensures a pulse energy of unity. Figure 3 plots one period of the pulse shape $h(t)$ in the time domain. The duration of the CI pulse shape is time limited to one period $T_{s}(576.6 \mu \mathrm{s}-30.44 \mu \mathrm{s}$ (guard time) in GSM) and not $T_{b}(3.69 \mu \mathrm{s}$ in GSM). To transmit a burst of bits, the $k^{t h}$ bit in a user's burst is modulated by the CI pulse shape $h\left(t-\tau_{k}\right)$, creating the total transmitted signal

$$
s(t)=\sum_{k=1}^{K} a_{k} h\left(t-\tau_{k}\right) \cdot g(t)
$$

where $a_{k}$ refers to the $k^{t h}$ data symbol and is binary antipodal; $g(t)$ refers to the rectangular function that extends over a slot duration $T_{s}$ (ensuring that the pulse shape does not extend beyond the user's allocated time slot); $\tau_{k}$ refers to the delay of the CI pulse shape associated with bit $a_{k}$, and $K$ refers to the total number of bits in a burst.

To determine the delays $\tau_{k}, k=1,2, \ldots, K$ and number of bits $(K)$ in a burst, the cross correlation (CC) function of the $\mathrm{CI}$ pulse shape is examined. The expression for the $\mathrm{CC}$ between the pulse shape of (5) delayed by time $t_{1}$ and the pulse shape delayed by time $t_{2}$ can be shown to be

$$
\begin{gathered}
R_{t_{1}, t_{2}}(\tau)=\frac{1}{2 \Delta f} \sum_{i=1}^{N} \cos (i(2 \pi \Delta f \tau)) \\
R_{t_{1}, t_{2}}(\tau)=\frac{1}{2 \Delta f} \cdot \cos 2 \pi \Delta f \tau \cdot \frac{\sin \left(\frac{1}{2} N 2 \pi \Delta f \tau\right)}{\sin \left(\frac{1}{2} 2 \pi \Delta f \tau\right)} \\
\cdot \cos \left(\frac{(N-1)}{2} 2 \pi \Delta f \tau\right)
\end{gathered}
$$

where $\tau$ denotes the relative time shift $t_{1}-t_{2}$.

This CC term demonstrates $2(N-1)$ zeros:

- $N-1$ equally spaced zeros at $\left\{\frac{p}{N \Delta f}=p T_{b}, p=\right.$ $1,2, \ldots, N-1\}$ resulting from the $\frac{\sin (\cdot)}{\sin (\cdot)}$ term, and

- $N-1$ equally spaced zeros at $\left\{\frac{2 p-1}{2(N-1) \Delta f}=\right.$ $\left.\frac{N(2 p-1)}{2(N-1)} T_{b}, p=1,2, \ldots, N-1\right\}$ as a result of the second $\cos (\cdot)$ term.

The existence of the first set of $N$ equally spaced zeros indicates that a CI/TDMA system can simultaneously support $N$ orthogonal pulse shapes in a slot by using the usual pulse shapes $h(t), h\left(t-T_{b}\right), \ldots, h\left(t-N T_{b}\right.$ ) (i.e., $\tau_{k}=k T_{b}$ for $k=1,2, \ldots, N)$. The existence of a second set of zeros, equally spaced by time separation $\frac{N}{(N-1)} T_{b}$, indicates that we can place an additional $(N-1)$ pulse shapes at highly (but pseudo) orthogonal locations. These pulses are positioned at $h\left(t-\frac{N}{2(N-1)} T_{b}\right), h\left(t-\frac{3 N}{2(N-1)} T_{b}\right), \ldots, h(t-$ $\left.\frac{N(2 N-3)}{2(N-1)} T_{b}\right)$ (i.e., $\tau_{k}=\frac{N}{2(N-1)} \cdot(2((k-N)-1)) T_{b}$ for $k=N+1, N+2, \ldots, K)$. The ability to position additional pulse shapes pseudo orthogonally in time (and modulate one bit or symbol on each pulse shape) provides the benefit of increased throughput in CI/TDMA systems. 


\section{Receiver Structures}

We assume a fading channel that is slow fading over each burst, and frequency selective over the entire bandwidth, $B W$, but flat over each frequency component that makes up the CI pulse shape (see Section 5). Hence, each frequency component making up the CI pulse shape experiences a different fade. The received signal is characterized by

$$
\begin{aligned}
r(t)= & \sum_{\substack{k=1 \\
\\
+\eta_{i}(t)}}^{K} a_{k} \sum_{i=1}^{N} \alpha_{i} A \cdot \cos \left(2 \pi i \Delta f\left(t-\tau_{k}\right)+\phi_{i}\right) \cdot g(t)
\end{aligned}
$$

where $\alpha_{i}$ is the gain and $\phi_{i}$ the phase offset in the $i^{t h}$ carrier of the CI pulse shape (due to the channel fade). To simplify the analysis, exact phase synchronization is assumed.

The CI/TDMA receiver detects the $j^{\text {th }}$ bit in the TDMA burst as shown in Figure 4 . Here, the $j^{\text {th }}$ bit is separated into its $N$ carrier components, outputting $\underline{\mathrm{r}}_{j}=$ $\left(r_{j, 1}, r_{j, 2}, \ldots ., r_{j, N}\right)$ where $r_{j, i}$ is the $i^{\text {th }}$ component and corresponds to

$$
\begin{aligned}
r_{j, i}= & \frac{1}{N} \alpha_{i} a_{j}+\sum_{l=1, l \neq j}^{K} \frac{1}{N} \alpha_{i} a_{l} \cos \left(2 \pi i \Delta f\left(\tau_{j}-\tau_{l}\right)\right) \\
& +\eta_{j, i}
\end{aligned}
$$

The second term represents the presence (in the $i^{\text {th }}$ carrier) of the other bits in a user's burst.

A suitable strategy must be found to combine the $r_{j, i}$ 's. Orthogonality restoring combining (ORC) involves the scaling of each $r_{j, i}$ by $\alpha_{i}$ (creating $r_{j, i} / \alpha_{i}$ ) and a summing of the terms (creating $R=\sum_{i=1}^{N} r_{j, i} / \alpha_{i}$ ). This enables the elimination of the orthogonal bits, but can result in substantial noise enhancement. Minimum mean square error combining (MMSEC) is a powerful alternative which attempts to jointly minimize the second term and the noise term. In our case, employing MMSEC results in the decision variable $R$ given by the linear sum

$$
R=\sum_{i=1}^{N} r_{j, i} \cdot\left[\frac{\alpha_{i}}{\left(P_{i} \alpha_{i}^{2}+N_{o}\right)}\right]
$$

where $P_{i}=\sum_{p=1}^{K} \cos \left(2 \pi \Delta f i\left(\tau_{j}-\tau_{p}\right)\right)^{2}$.

In MMSEC, note that for small $\alpha_{i}$, the gain avoids excessive noise amplification, while for large $\alpha_{i}$, the gain becomes proportional to the inverse of the subcarrier envelope, in order to recover the available orthogonality among pulse shapes.

\section{Channel Model}

The multipath fading channel model used to assess the performance of the CITDMA system is taken from the
COST-207 GSM system standard[7]. Here a series of channel models such as the hilly terrain (HT) and typical urban (TU) models are defined as transversal filters with time varying coefficients. The average power of the coefficients is determined by the multipath power delay profile (PDP), and an example is provided in Table 1. For realistic vehicle speeds, the coherence time of this channel is greater than the duration of a time slot - hence, the channel is considered constant during the transmission of a burst (but varies from one user's burst to the next).

In typical GSM/TDMA simulations, the PDPs characterize the ISI introduced by the channel. In CI/TDMA, where pulse shapes consist of multiple carrier transmissions which are frequency separated at the receiver, the channel must be characterized by $(\Delta f)_{c}$, the coherence bandwidth (defined as the bandwidth over which the frequency correlation function is above 0.5 ). This is computed from multipath PDP by using the relationship [1]

$$
(\Delta f)_{c} \approx \frac{1}{5 \sigma_{\tau}}
$$

where $\sigma_{\tau}$ is the rms delay spread and is computed according to

$$
\begin{gathered}
\sigma_{\tau}=\sqrt{\tau^{\prime}-(\tau)^{2}} \\
\tau=\frac{\sum_{k} \alpha_{k}^{2} \tau_{k}}{\sum_{k} \alpha_{k}^{2}} \\
\tau^{\prime}=\frac{\sum_{k} \alpha_{k}^{2} \tau_{k}^{2}}{\sum_{k} \alpha_{k}^{2}}
\end{gathered}
$$

Here, $\alpha_{k}$ is the power of the multipath component arriving at delay $\tau_{k}$. This leads to, e.g., a coherence bandwidth of $39.72 \mathrm{KHz}$ and $188 \mathrm{KHz}$ for the HT and TU channel respectively. For both HT and TU channels, as is typical in most mobile environments, the $(\Delta f)_{c}$ value satisfies

$$
1 / T_{s}<<(\Delta f)_{c}<B W
$$

where $B W=1 / T_{b}$ is the total bandwidth of the system. Equation (17) indicates that the mobile channel is frequency selective over the entire bandwidth of transmission, but not over each subcarrier[5]. Specifically, with $N$ carriers residing over the entire bandwidth, $B W$, each carrier undergoes a flat fade, with the correlation between the $i^{\text {th }}$ subcarrier fade and the $j^{\text {th }}$ subcarrier fade characterized by [8]

$$
\rho_{i, j}=\frac{1}{1+\left(\left(f_{i}-f_{j}\right) /(\Delta f)_{c}\right)^{2}}
$$

where $\left(f_{i}-f_{j}\right)$ indicates the frequency separation between the $i^{\text {th }}$ and the $j^{\text {th }}$ subcarriers. Generation of fades with correlation has been discussed in [9]. 


\section{Performance Results}

Figure 5 presents bit error probability (BER) versus SNR performance curve for the HT channel. The bottom (dashdot) curve represents the CI/TDMA system with 148 orthogonal pulse shapes per slot and one bit modulated on each pulse shape. The dashed line marked with stars shows the performance of the CI/TDMA system when 146 more bits are added using pseudo orthogonal positioning of $\mathrm{CI}$ pulse shapes. The solid line represents the benchmark GSM system (with $N=148$ bits per slot). The benchmark GSM system corresponds to typical Gaussian GSM pulse shaping with the DFE receiver of [2]. Figure 5 clearly shows that the new CITDMA scheme with 148 bits achieves up to $8 \mathrm{~dB}$ gain in probability of errors, where BER is in the order of $10^{-2}$ (more significant gains are achievable with smaller probability of error). Even with 146 additional bits, the CI/TDMA system outperforms the DFE receiver by 6.5 $\mathrm{dB}$ at BER of $10^{-2}$. Furthermore, the $\mathrm{CI} / \mathrm{TDMA}$ combining receiver is comparable to the DFE in complexity.

Similar results are shown in Figure 6 for the TU channel. The CITDMA system provides a $5 \mathrm{~dB}$ gain with 148 bits in one TDMA time slot. With 294 bits in a slot the CI/TDMA system provides up to $4 \mathrm{~dB}$ gain. The benchmark is again the GSM system with DFE receiver of [2].

These results also show that energy harnessed from a frequency domain combining (creating frequency diversity benefits) provides greater benefit than the time domain based equalizer structures. CI/TDMA provides an efficient way of exploiting the frequency domain benefits through pulse shaping. Since it retains all the features of a TDMA system, the higher protocol levels currently in use for TDMA systems are equally applicable to the CI/TDMA systems.

\section{Conclusions}

In this work a novel pulse shaping method that involves carrier interferometry is investigated and is shown to support a TDMA architecture. The ability to overlap the pulse shapes orthogonally as well as pseudo orthogonally enables the CI/TDMA system to support greater throughputs with no expense in bandwidth. The multi-carrier pulse shaping does not require an equalizer at the receiver. Instead, using suitable combining strategies at the receiver, the system exploits frequency diversity benefits that are inherent in such multi-carrier systems. Simulation results show an increase of nearly $100 \%$ in throughput when compared to that of a GSM system employing a conventional DFE. These results were obtained with gains in performance of up to $6.5 \mathrm{~dB}$ relative to the benchmark GSM system at BER of $10^{-2}$.
[1] T.S.Rappaport, Wireless Communications - Principles and Practice. New Jersey: Prentice Hall, $1^{\text {st }}$ ed.,1996.

[2] Bjorn A.Bjerke, J.G.Proakis, M.K.Lee and Z.Zvonar, "A comparison of Decision feedback equalization and data directed Estimation technique for the GSM system,"IEEE 6th International conference on Universal personal communications, 1997

[3] Tijdhof.J.J.H et al., "On the design and realization of adaptive equalization for mobile communication," First IEEE signal processing workshop on signal processing advances in wireless communications, April 16-18, Paris, France 1997.

[4] G.D.Aria, R.Piermarini and V.Zingarelli, "Fast Adaptive Equalizers for narrowband TDMA Mobile Radio," IEEE Trans. on Vehic. Tech.,pg 392-404, May 1991.

[5] J.Proakis, Digital Communications. New York: McGraw-Hill, $3^{\text {rd }}$ ed., 1995.

[6] P.A.Laurent,"Exact and approximate construction of digital phase modulations by superposition of amplitude modulated pulses," IEEE Trans. on Communication, pages 150-160, Feb. 1986.

[7] COST-207: "Digital land mobile radio communications," Final report of the COST-Project 207, Commission of the European Community, Brussels, 1989.

[8] W.Xu and L.B.Milstein, "Performance of Multicarrier DS CDMA Systems in the presence of correlated fading," IEEE $47^{\text {th }}$ Vehicular Technology Conference, Phoenix, AZ, May 4-7, 1997, pp. 2050-4

[9] B.Natarajan, C.R.Nassar and V.Chandrasekhar, "Generation of Correlated Rayleigh Fading envelopes for spread spectrum applications,"IEEE Communication letters, vol.4, no. 1, pp.9-11,January 2000.

\begin{tabular}{|c|c|c|}
\hline \multicolumn{3}{|c|}{ Hilly Terrain } \\
\hline Tap No. & $\tau(\mu s)$ & $\alpha(\mathrm{dB})$ \\
\hline 1 & 0.0 & 0.0 \\
\hline 2 & 0.2 & -2.0 \\
\hline 3 & 0.4 & -4.0 \\
\hline 4 & 0.6 & -7.0 \\
\hline 5 & 15.0 & -6.0 \\
\hline 6 & 17.2 & -12.0 \\
\hline
\end{tabular}

Table 1: Multipath Power Delay Profiles for the HT channel

\section{References}




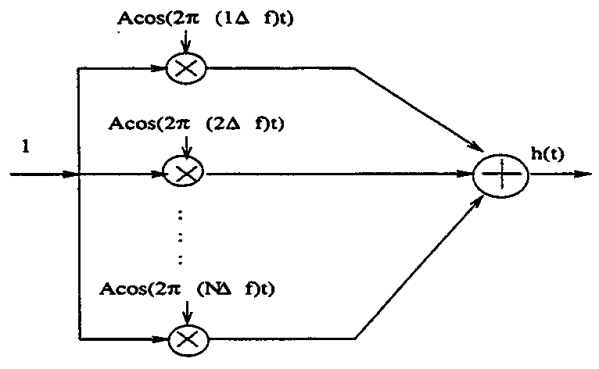

Figure 1. Generation of Cl Pulse shape

(a)

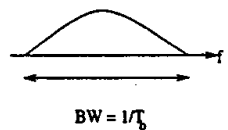

(b)

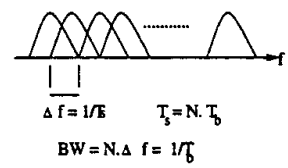

Figure 2. Frequency domain representations (a) GSM system (b) CI/TDMA system

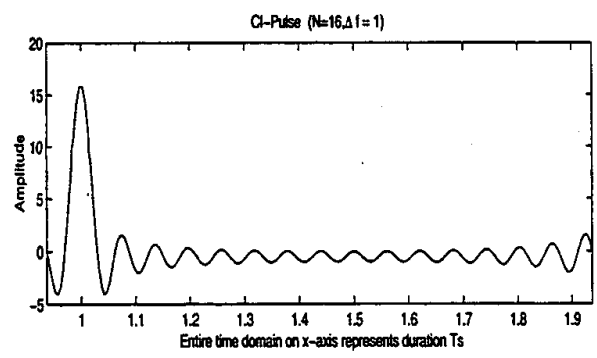

Figure 3. Cl Pulse shape (note that a time delay of ' 1 ' is introduced for ease in presentation)

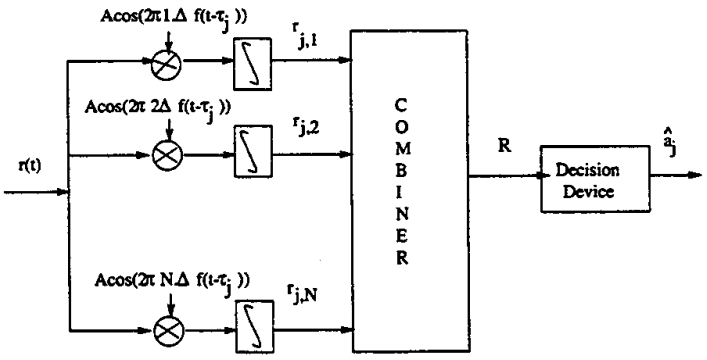

Figure 4. CI/TDMA Receiver structure

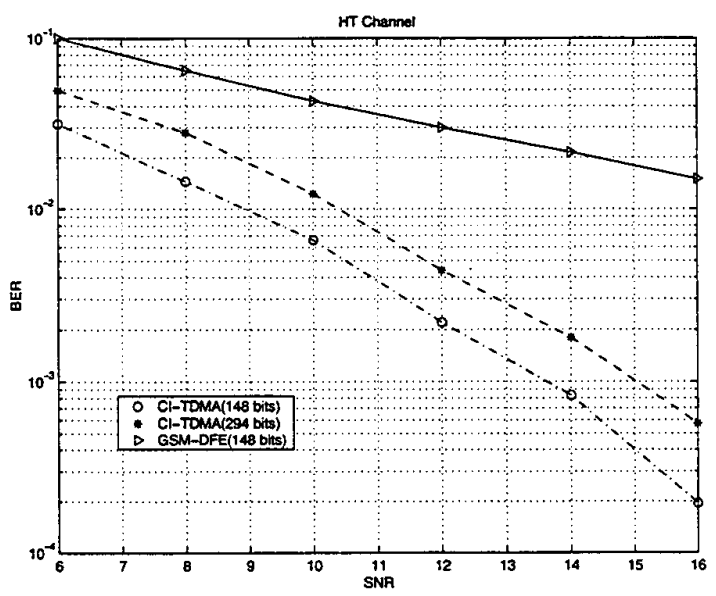

Figure 5. BER comparison in HT channel

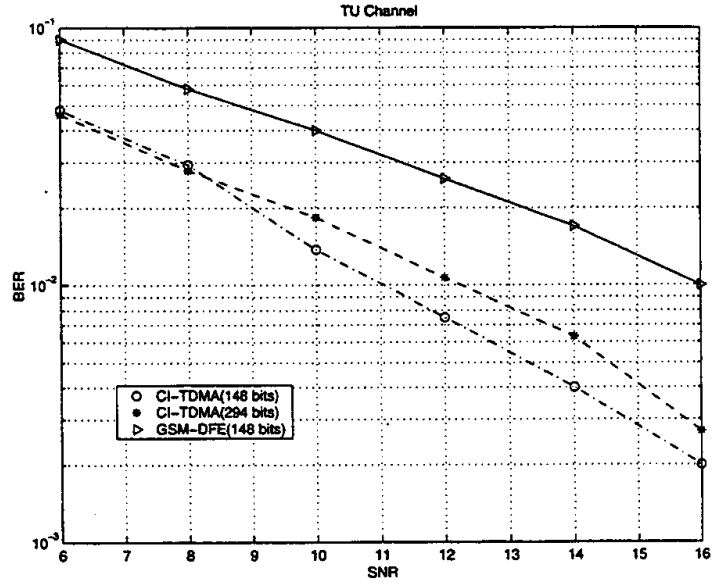

Figure 6. BER comparison in TU channel 